\title{
Modified silica ceramic for frequency selective surface radome
}

\author{
Long-Long ZHANG, Ji-Hua ZHANG, ${ }^{\dagger}$ Xiao-Lang CHEN, Hong-Wei CHEN and Chuan-Ren YANG \\ State Key Laboratory of Electronic Thin Films and Integrated Devices, University of Electronic Science and Technology of China, \\ Chengdu 610054, Sichuan, PR China
}

\begin{abstract}
Frequency selective surface (FSS) based on ceramic materials is very promising due to its high temperature bearing capacity along with stealth properties for high-speed aircraft. In this paper, modified silica ceramic, which is compatible for High Temperature Co-fired Ceramic (HTCC) technology, was investigated. The flexural strength of the $\mathrm{Li}_{2} \mathrm{O}-\mathrm{K}_{2} \mathrm{O}-\mathrm{Al}_{2} \mathrm{O}_{3}$ doped silica ceramic reaches $163.65 \mathrm{MPa}$ at a sintering temperature of $1325^{\circ} \mathrm{C}$, which greatly surpasses that of conventional fused silica. The maximum density of the ceramic is $2.113 \mathrm{~g} / \mathrm{cm}^{3}$. The dielectric constant is 4.37 and the dissipation factor is 0.0038 at $14 \mathrm{GHz}$. Based on the modified silica ceramic, a Jerusalem element FSS was designed and optimized. Results show that the Jerusalem element FSS owes its advantages to high transmission coefficient, appropriate bandwidth, flat pass band and stability with different polarizations.
\end{abstract}

(C2015 The Ceramic Society of Japan. All rights reserved.

Key-words : Silica ceramic, Frequency selective surface, Radome, Dielectric properties

[Received January 6, 2015; Accepted July 13, 2015]

\section{Introduction}

Radome stealth technology is one of the focuses of current stealth technologies. Radar antenna systems consist of radar antennas, radome and high-frequency components of the radar cabin and a strong scattering source in the nose region of an aircraft. Its stealth effect has a direct impact on the aircraft's stealth effect. ${ }^{1)}$ There are two ways to reduce an aircraft radar cross section (RCS) at present: One is a low RCS shape design and the other is radar absorbing coating technology. In order to reduce RCS as much as possible, a combination of the two approaches is often used by researchers. ${ }^{2)}$ However, due to the fact that the radar antenna system needs to ensure the normal transmission and reception of radar waves, the stealth methods mentioned above cannot easily be applied to antenna stealth. ${ }^{3), 4)}$ Therefore, radar antenna system stealth becomes a bottleneck of aircraft stealth. Currently, the most widely used technique is FSS for radome stealth. ${ }^{2), 5), 6)}$

FSS is any surface construction designed as a "filter" for plane waves. This surface is comprised of periodically arranged metallic-patch elements or aperture elements within a metallic screen, which exhibit total reflection or transmission characteristics respectively in the neighborhood of the element resonance. ${ }^{7), 8)}$ However, conventional FSS radomes based on organic materials are difficult to apply in high speed aircraft because of their relatively low applied temperature. So, an FSS radome based on inorganic ceramic with high temperature tolerance needs to be developed. Conventional ceramic radomes without stealth function are already in extensive use, but due to confidential reasons, there are few reports which show the research on ceramic FSS radomes. The ceramic materials used in hypersonic aircraft radomes are silica, boron nitride and silicon nitride ceramics. ${ }^{9-16)}$ Fused silica has generally been used as such a material for radomes due to its high chemical stability, low dielectric constant and loss tangent. ${ }^{9)}$ However, the low strength

$\uparrow$ Corresponding author: J.-H. Zhang; E-mail: jhzhang@uestc. edu.cn cannot meet the requirements for hypersonic aircraft. Introducing fine-scale fibers can improve the mechanical strength of fused silica, but the dielectric property is impaired. ${ }^{17)}$ Therefore, it is important to keep mechanical and dielectric properties balanced to meet practical application requirements.

In this paper, fused silica was used as a matrix for the dielectric material of a ceramic FSS radome. In order to obtain high bending strength and compatibility with HTCC technology of ceramic FSS, but not impair its dielectric properties significantly, $\mathrm{Li}_{2} \mathrm{O}-$ $\mathrm{K}_{2} \mathrm{O}-\mathrm{Al}_{2} \mathrm{O}_{3}$ was doped into the fused silica. The results indicate that the bending strength of the fused silica doped with $\mathrm{Li}_{2} \mathrm{O}-$ $\mathrm{K}_{2} \mathrm{O}-\mathrm{Al}_{2} \mathrm{O}_{3}$ has been greatly improved and the sintering temperature has adjusted to allow for metal-ceramic co-fire. Based on the modified fused silica dielectric, a dual screen bandpass ceramic FSS radome was designed.

\section{Experimental procedures and results}

\subsection{The properties of pure silica}

According to the crystal phase transition theory of silica ceramic, the sintering temperature of pure silica we selected was between 1100 and $1300^{\circ} \mathrm{C}$. Figure 1 shows the XRD profiles of pure silica achieved from different sintering temperatures and

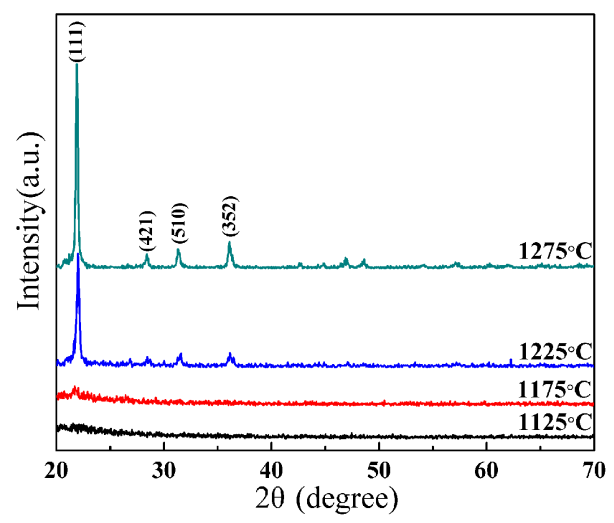

Fig. 1. The XRD profiles of pure silica. 

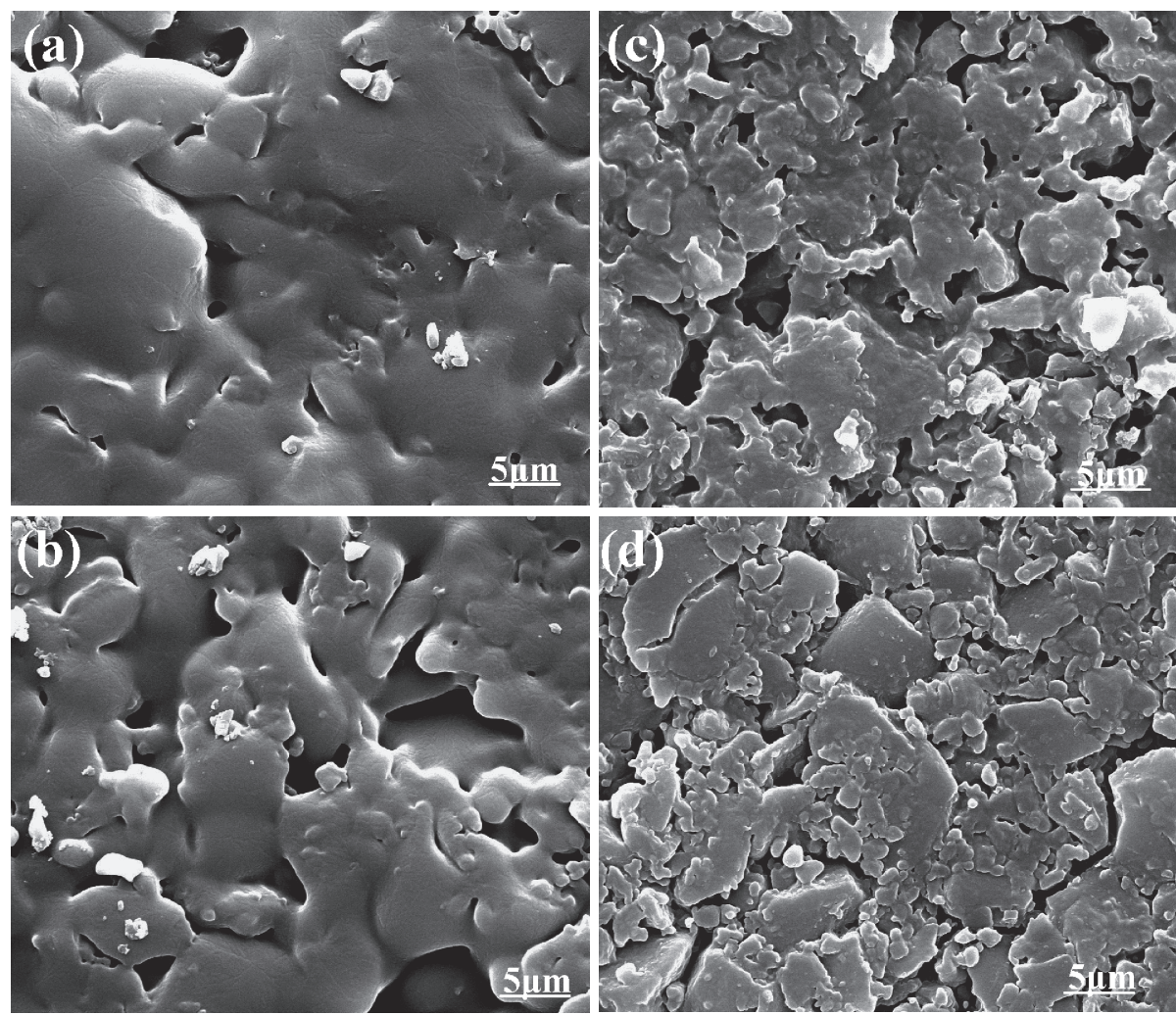

Fig. 2. Surface morphology of pure silica: (a) $1125^{\circ} \mathrm{C}$; (b) $1175^{\circ} \mathrm{C}$; (c) $1225^{\circ} \mathrm{C}$; (d) $1275^{\circ} \mathrm{C}$.

Fig. 2 shows its SEM photos. It can be seen that when the sintering temperature is low, there are no obvious diffraction peaks, and the samples are amorphous. With the increase of sintering temperature, diffraction peaks appear gradually, and crystallinity increases gradually.

Figure 3(a) shows the flexural strengths and densities of pure silica in different sintering temperatures. It can be known that the flexural strengths are less than $16 \mathrm{MPa}$ and the densities are less than $2.2 \mathrm{~g} / \mathrm{cm}^{3}$. Figure 3(b) shows the microwave dielectric properties of pure silica measured at $14 \mathrm{GHz}$ by resonance cavity method. The results show that the dielectric constant of pure silica varies from 2.68 to 3.77 and the dissipation factor varies from 0.0041 to 0.0092 when the sintering temperature increases from 1125 to $1275^{\circ} \mathrm{C}$. In general, pure silica has good microwave dielectric properties and low density. But its flexural strength is too low, so it cannot meet the requirements for hypersonic aircraft. In order to increase the flexural strength of silica ceramic, we try to implement a doping modification on it.

\subsection{The preparation of doped silica ceramic}

In our study, the doped silica ceramic was obtained through a high temperature solid-state reaction sintering method. The raw materials we used were $\mathrm{SiO}_{2}, \mathrm{Al}_{2} \mathrm{O}_{3}, \mathrm{~K}_{2} \mathrm{CO}_{3}$ and $\mathrm{Li}_{2} \mathrm{CO}_{3}$. The platinum electrode we used as the metal layer of ceramic FSS radome was from the Kunming Institute of Precious Metals of China, and its upper limit of sintering temperature was $1460^{\circ} \mathrm{C}$. In order to improve the flexural strength of the fused silica, $\mathrm{Al}_{2} \mathrm{O}_{3}, \mathrm{~K}_{2} \mathrm{CO}_{3}$ and $\mathrm{Li}_{2} \mathrm{CO}_{3}$ were used as sintering additives. The sintering temperature was controlled between 1300 and $1400^{\circ} \mathrm{C}$. The mass fractions of raw materials in the samples were $86.33 \%$ $\mathrm{SiO}_{2}, 3.38 \% \mathrm{Al}_{2} \mathrm{O}_{3}, 6.41 \% \quad \mathrm{~K}_{2} \mathrm{CO}_{3}$ and $3.84 \% \quad \mathrm{Li}_{2} \mathrm{CO}_{3}$. The mechanical property, density and dielectric properties of the samples were measured.
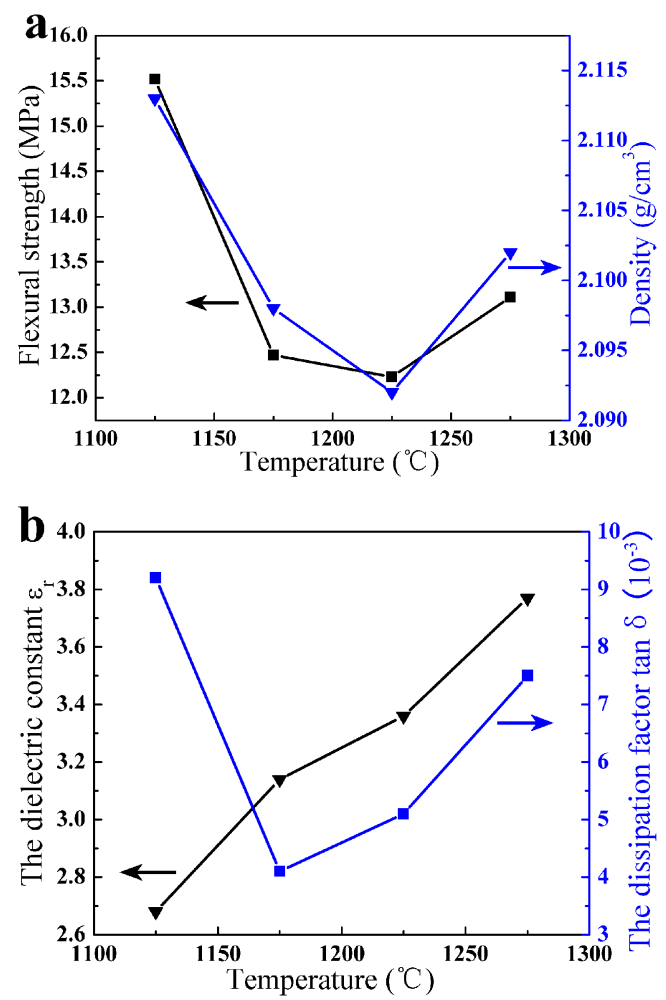

Fig. 3. (a) The flexural strength and density of pure silica; (b) The dielectric properties of pure silica. The measured frequency is $14 \mathrm{GHz}$.

\subsection{Experimental results and discussion}

Figure 4 shows the XRD profiles of the samples achieved from different sintering temperatures. It can be seen that when the 
sintering temperature is low, the main phase of the sample is tridymite and there are no more prominent peaks. As the sintering temperature increases, the intensity of diffraction peaks of the tridymite phase weakens. At the same time, new diffraction peaks appear at the diffraction angles near 28 and $32^{\circ}$, which indicate that a new substance is generated. Compared with standard PDF cards, the new substance is $\mathrm{LiAlSi}_{2} \mathrm{O}_{6}$ and it only appears when the sintering temperature is above $1350^{\circ} \mathrm{C}$. $\mathrm{LiAlSi}_{2} \mathrm{O}_{6}$ 's emergence will reduce the flexural strength of the samples because the content of sintering additives melt into glass phase decreases.

The SEM photos of the samples in different sintering temperatures are shown in Fig. 5. As can be seen from the figure, at $1300^{\circ} \mathrm{C}$, the doped silica ceramic's surface has minor undulations as well as white particles and spots; these may be impurities which do not melt sufficiently. As the sintering temperature gradually increases, the samples melt into a further glassy state,

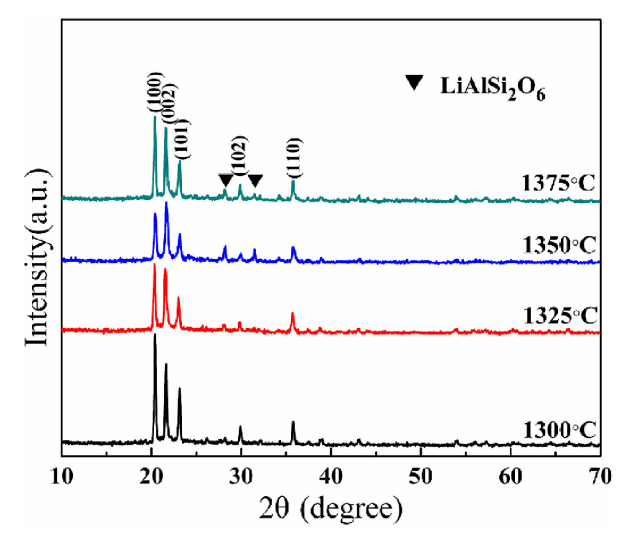

Fig. 4. The XRD profiles of the doped silica. the surfaces become smoother and the melting transition becomes complete. The surfaces of the samples are smooth without voids, pores or cracks and they largely melt into a glassy state with a very dense structure, therefore, the flexural strength of silica ceramic is expected to be improved compared with fused silica.

Figure 6(a) shows the flexural strengths and densities of the samples in different sintering temperatures. It can be known that the flexural strength varies from 146.58 to $163.65 \mathrm{MPa}$ and the density varies from 2.092 to $2.113 \mathrm{~g} / \mathrm{cm}^{3}$. As expected, $\mathrm{LiAlSi}_{2} \mathrm{O}_{6}$ 's emergence reduces the flexural strength by a small amount. Flexural strength and density have the same changing trend. The flexural strength has greatly improved compared with that of fused silica of $43 \mathrm{MPa}$ but the density is only slightly different to that of fused silica of $2.2 \mathrm{~g} / \mathrm{cm}^{3}{ }^{3}{ }^{\text {9) }}$ As sintering additives, $\mathrm{Al}_{2} \mathrm{O}_{3}, \mathrm{~K}_{2} \mathrm{CO}_{3}$ and $\mathrm{Li}_{2} \mathrm{CO}_{3}$ not only reduce the sintering temperature, but also convert into liquid glass phase in the sintering process. The liquid glass, which bonds silica particles together, leads to a dense structure, thus greatly improving the flexural strength.

At the resonant frequency of about $14 \mathrm{GHz}$, the microwave dielectric properties change with the sintering temperature as shown in Fig. 6(b). Results show that the dielectric constant of the ceramic varies from 4.37 to 4.58 and the dissipation factor varies from 0.0038 to 0.0053 when the sintering temperature increases from 1300 to $1375^{\circ} \mathrm{C}$. The dielectric constant and the dissipation factor have similar changing trends and the dielectric properties are best at $1350^{\circ} \mathrm{C}$. The dielectric constant and dissipation factor of fused silica are 3.42 and $0.0004 .{ }^{9)}$ Compared with fused silica, the dielectric properties of doped silica ceramic have deteriorated, but they are still within an acceptable range. ${ }^{9)}$ It can be seen that the glass modified ceramic possesses the appropriate flexural strength, density and dielectric properties as a radome material.
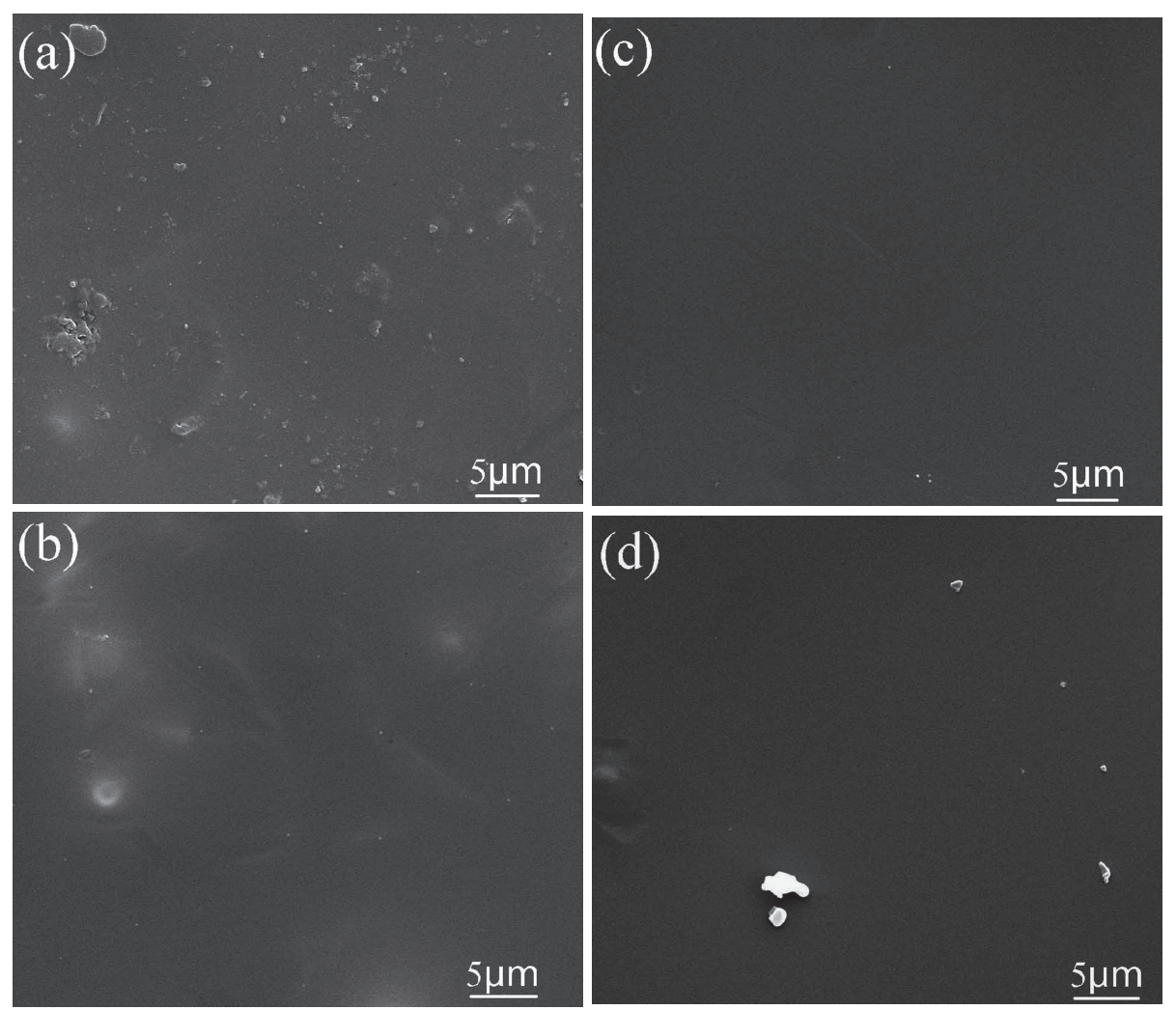

Fig. 5. Surface morphology of doped silica ceramic: (a) $1300^{\circ} \mathrm{C}$; (b) $1325^{\circ} \mathrm{C}$; (c) $1350^{\circ} \mathrm{C}$; (d) $1375^{\circ} \mathrm{C}$. 


\section{Design of FSS radome based on the modified ceramic}

In order to verify that ceramic can be used as the material of FSS radome in theory, we then used it as the dielectric layer in
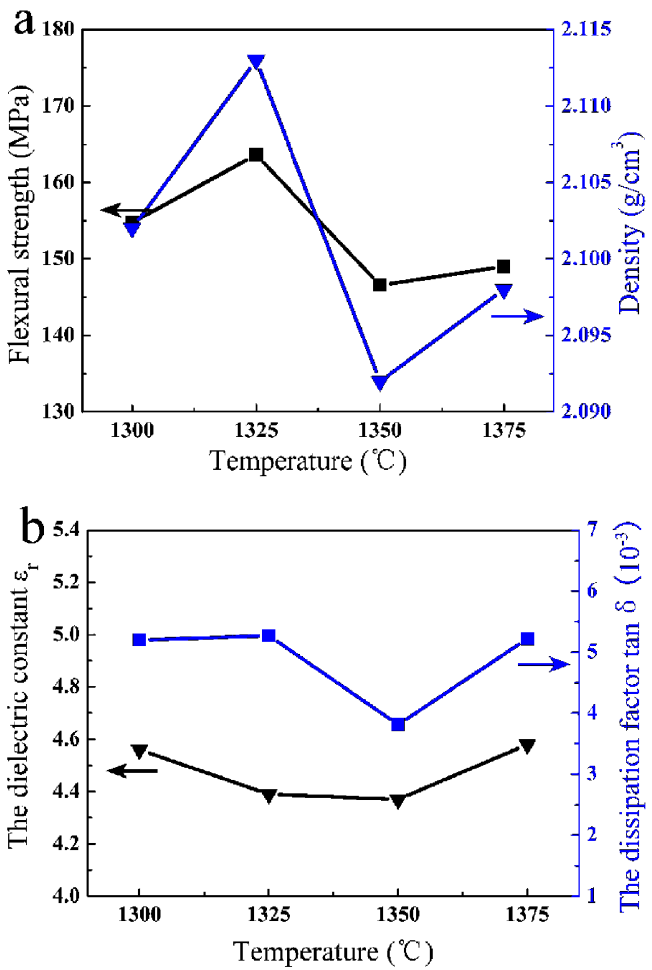

Fig. 6. (a) The flexural strength and density of the ceramic; (b) The relationship between the dielectric properties and sintering temperature. the design of a ceramic FSS radome. It is well known that Jerusalem FSS has good frequency characteristics, especially the center frequency of the pass band, which does not change with the change of incidence angle. ${ }^{18)}$ Therefore, in our design, a Jerusalem FSS was simulated in CST Microwave Studio.

A Jerusalem element FSS is described in Fig. 7(a). The gray part is metal platinum and the white part is the aperture. The dielectric loading mode is shown in Fig. 7(b). The material of the dielectric layers is the silica ceramic we have obtained, which has a dielectric constant set at 4.37 and a dissipation factor set at 0.0038 . In the model, the boundary conditions are set as: Set two Floquet ports at the $\mathrm{Z}$ direction as the incident and exit ports of electromagnetic waves, $\mathrm{X}$ and $\mathrm{Y}$ directions are set as unit cell, $\mathrm{Z}$ direction is open (add space). Then we use the frequency solver in CST Microwave Studio to calculate the model's frequency response characteristics.

Figure 7(c) shows the frequency response of Jerusalem FSS at normal incidence as obtained from full-wave EM simulations in CST Microwave Studio. As can be seen from the figure, for TE polarization, the center frequency is $9.075 \mathrm{GHz}$, in-band insertion loss is $0.604 \mathrm{~dB},-1 \mathrm{~dB}$ bandwidth is $1.177 \mathrm{GHz}$ and for TM polarization, the center frequency is $9.06 \mathrm{GHz}$, in-band insertion loss is $0.56 \mathrm{~dB},-1 \mathrm{~dB}$ bandwidth is $1.185 \mathrm{GHz}$. Thus, the center frequency, transmittance and $-1 \mathrm{~dB}$ bandwidth of Jerusalem FSS for different polarization of incident electromagnetic wave has good stability. As shown in Fig. 7(c), the reflection coefficient S21 and transmission coefficient S11 curves of TE and TM polarizations are very similar. The small insertion loss of about $0.6 \mathrm{~dB}$, which is mainly contributed by the loss of dielectric, indicated that our modified ceramic can fit the requirements of FSS. Of course, as a filter, the Jerusalem FSS does not have ideal out-of-band rejection performance for either TE or TM polarized incident wave. It may need further optimize in the design of the FSS element.
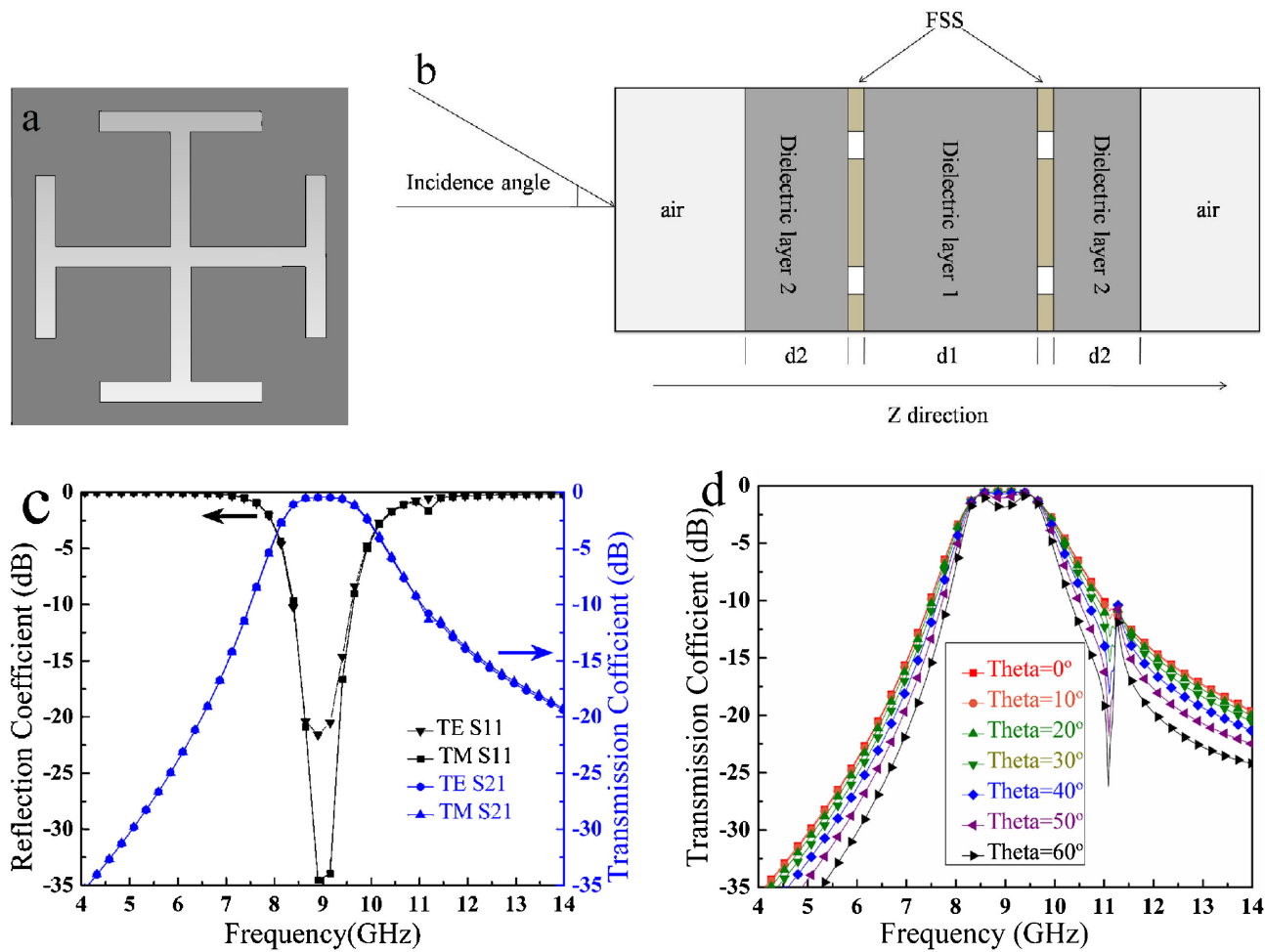

Fig. 7. (a) Jerusalem element; (b) Dielectric loading mode of the dual screen ceramic FSS radome; (c) The simulated results of Jerusalem FSS at normal incidence; (d) The change of S21 with the change of the incidence angle for TE polarization. 
The stability of incidence angle is the key performance of FSS. Figure 7(d) shows the changing curves of S21 of Jerusalem FSS with the change of incidence angle for TE polarization. The figure shows that as the incidence angle of the TE wave increases, transmission coefficient decreases, in-band insertion loss increases, and pass band flatness becomes worse. When the incident angle is $50^{\circ}$, the in-band insertion loss is significantly increased, so the stability of the incident angle can be maintained around $40^{\circ}$.

In summary, Jerusalem FSS based on our glass modified silica ceramic has a high transmission coefficient, appropriate bandwidth and a flat pass band, so the ceramic we have obtained can be used as the dielectric material of a FSS radome.

\section{Conclusions}

Fused silica doped with $\mathrm{Li}_{2} \mathrm{O}-\mathrm{K}_{2} \mathrm{O}-\mathrm{Al}_{2} \mathrm{O}_{3}$ was fabricated as a radome material by the high temperature solid-state reaction sintering method. The flexural strength of the ceramic can reach 163.65 MPa. The dielectric constant of the ceramic is 4.37 and the dissipation factor is 0.0038 at the resonant frequency of about $14 \mathrm{GHz}$ when the sintering temperature is $1350^{\circ} \mathrm{C}$. Based on the dielectric characteristics of the doped silica, a Jerusalem FSS was designed. It was found that the Jerusalem FSS has high transmission coefficient, appropriate bandwidth, flat pass band and stability with different polarizations. These results reveal that the modified silica ceramic might be suitable for a stealth ceramic radome.

\section{References}

1) H. Y. Jia, J. S. Gao, X. G. Feng and L. C. Sun, Acta Phys. Sin., 58, 505-510 (2009).

2) G. W. Lu, J. Zhang, J. Y. Yang, T. X. Zhang and Y. Kou, Acta Phys. Sin., 62, 198401 (2013).
3) P. C. Kim, D. G. Lee, W. Lim and I. S. Seo, Compos. Struct., 90, 242-246 (2009).

4) P. C. Kim, D. G. Lee, I. S. Seo and G. H. Kim, Compos. Sci. Technol., 68, 2163-2170 (2008).

5) B. A. Munk, "Frequency Selective Surfaces: Theory and Design", 1st edition, Wiley-Interscience, New York (2000).

6) B. Widenberg, Thick Frequency Selective Structures, Ph.D. thesis, Lund Institute of Technology, Department of Electroscience, Lund Institute of Technology, P.O. Box 118, S-221 00 Lund, Sweden (2003) ISSN 1402-8662.

7) J. C. Vardaxoglou, "Frequency Selective Surfaces: Analysis and Design", Research Studies Press Ltd., Taunton (1997).

8 Z. L. Wang, K. Hashimoto, N. Shinohara and H. Matsumoto, IEEE Trans. Microw. Theory Tech., 47, 2039-2042 (1999).

9) G. J. Qi, C. R. Zhang, H. F. Hu, F. Cao, S. Q. Wang, Y. B. Cao and Y. G. Jiang, J. Chin. Ceram. Soc., 33, 632-638 (2005).

10) S. Q. Li, Y. C. Pei, C. Q. Yu and J. L. Li, Ceram. Int., 35, 1851-1854 (2009).

11) F. Chen, F. Cao, H. L. Pan, K. Y. Wang, Q. Shen, J. L. Li and S. Q. Wang, Mater. Des., 40, 562-566 (2012).

12) R. B. Zhang, D. N. Fang, Y. M. Pei and L. C. Zhou, Ceram. Int., 38, 4373-4377 (2012).

13) R. B. Zhang, D. N. Fang, X. M. Chen and Y. M. Pei, Ceram. Int., 38, 6021-6026 (2012).

14) Y. Inagaki, N. Kondo and T. Ohji, J. Eur. Ceram. Soc., 22, 2489-2494 (2002).

15) J. H. She, J. F. Yang, D. D. Jayaseelan, N. Kondo, T. Ohji, S. Kanzaki and Y. Inagaki, J. Am. Ceram. Soc., 86, 738-740 (2003).

16) Y. Zhang, Y. B. Cheng, S. Lathabai and K. Hirao, J. Am. Ceram. Soc., 88, 114-120 (2005).

17) H. L. Du, Y. Li and C. B. Cao, J. Alloy. Compd., 503, L9-L13 (2010).

18) R. Mittra and D. Lee, IEEE Antennas Propag. Soc. Int. Symp., 4, 370-373 (2001). 